\title{
High Sensitive FBG Based Muscular Strain Sensor
}

\author{
R. D. Koozehkanani ${ }^{1}$, S. Makouei ${ }^{{ }^{*}}$ \\ ${ }^{1}$ Faculty of Electrical and Computer Engineering, University of Tabriz, Tabriz 51664, Iran \\ *corresponding author, E-mail: makouei@tabrizu.ac.ir
}

\begin{abstract}
Assessment of biomechanical behavior of human musculoskeletal structure is essential to recognize bone diseases and to design proper medical devices. The skeleton system basically adapts to mechanical loadings. Thus, monitoring the bone deformation under load is of great importance to attain better analysis and interpretation. In recent years, Fiber Bragg Grating sensing devices have been developed and used to monitor strain and temperature of skeleton system. In this work a Fiber Bragg Grating sensor is designed holding a $1.54 \mathrm{pm}^{-1} \varepsilon^{-1}$ axial strain sensitivity which is almost $30 \%$ higher than the one achieved so far. The improvement in sensitivity is achieved by adjusting single-mode optical fiber parameters of the structure.
\end{abstract}

\section{Introduction}

Generally, physical activity appears to play an important role in maximizing bone mass during childhood and the early adult years. Figure 1 shows different types of physical activities and corresponding maximum level of presented strains applied to human skeletal system. Admittedly, benefits of physical activity on bone health is better judged through determining the correlation of activity level with bone mass in a specific training program. Therefore, it is important to monitor the stress distribution along the skeletal structure during the growth and the whole lifetime so as to provide valuable information for maintenance purposes and fracture prevention and serious damage $[1,2]$. Among the various types of sensing elements, fiber Bragg Gratings (FBGs), due to their outstanding features, have exhibited a great potential for applications in biomechanics and rehabilitation engineering field [3-8]. The main advantages are small size, biocompatibility, high sensitivity, high bandwidths and multiplexing capabilities. Furthermore, immunity to electromagnetic and RF interferences, chemicals, radioactivity, corrosion and lightning is a remarkable characteristic of these elements. The mentioned features make a sensor suitable for long-term monitoring in human body uses. Studies towards synergizing FBG optical sensing technology with biomechanics and rehabilitation applications are very recent. They measure a wide range of parameters, such as strain inside and on the surface of bones, stress in bone cement during polymerization, pressure in orthopedic joints, tension in the intervertebral discs, pressure distribution on the Human machine interface, the dental biomechanics of tendons and ligaments in the angle between the different parts of the body, and many others [3-7]. In [9] new type of optical fiber strain sensor based on mode field diameter value modulation is presented. The structure is high sensitive and also has linear behavior.

The work presented in this article is a part of a project which seeks to develop a garment-integrated wearable bone deformation monitor for use during the whole lifetime. The paper focuses on the development of the FBG strain sensor in an initial structure prototype for high performance. The remainder of the paper is organized as follows. Section 2 presents FBG principles. In section 3 the initial FBG structure for strain sensor is introduced based on standard telecommunication step index single-mode optical fiber. Section 4 studies the adjustment of grating and structural parameters observing side lobe suppression and reflectivity response of sensor. Discussions and proposed FBG Sensor are presented in section 5 and finally the conclusion is demonstrated.

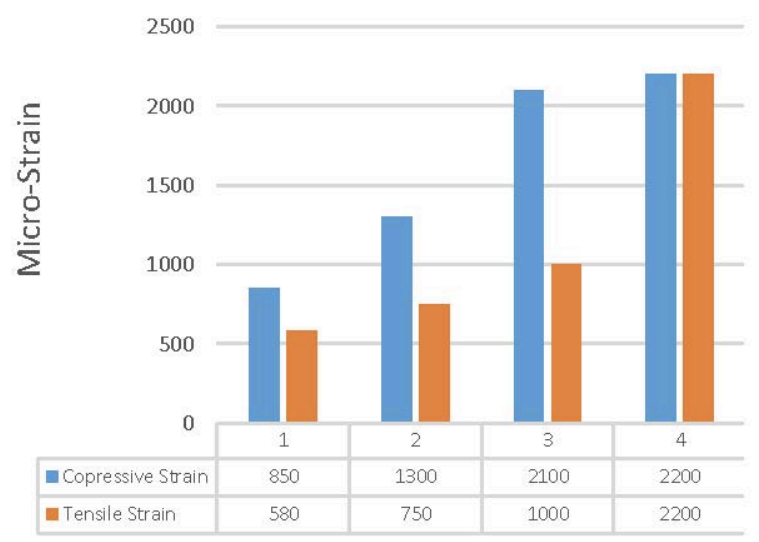

Figure 1: Adaption of skeletal system to strain or mechanical loads [1]

\section{Fiber Bragg Grating Principle}

A Fiber Bragg Grating is formed by modulating refractive index of an optical fiber periodically as described in figure 2. If a broadband light source is coupled into an optical fiber containing FBG part, the light with wavelength $\lambda_{B}$ called Bragg wavelength, is selectively reflected. The $\lambda_{B}$ is proportional to the grating period $\Lambda$ and the effective refractive index $n_{\text {eff }}$ and is defined by: 


$$
\lambda_{B}=2 n_{e f f} \Lambda
$$

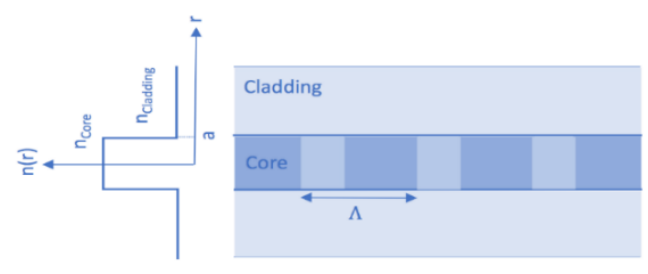

Figure 2: Fiber Bragg grating schematic

Any variation in $\Lambda$ and $n_{\text {eff }}$ leads to a shift in the $\lambda_{B}$. This principle is widely used in FBG based fiber optic sensors. It is evident that $\Lambda$ and $\mathrm{n}_{\text {eff }}$ depend on the strain applied to the grating part and to the temperature variation that grating part experiences. The sensing principle in FBG is based on the demodulation of brag wavelength modulation for measurement of the strain and temperature (figure 3). The shift in Bragg wavelength $\lambda_{\mathrm{B}}$ can be expressed as:

$$
\Delta \lambda_{\mathrm{B}}=2\left[\Lambda \frac{\partial n e f f}{\partial \varepsilon}+n e f f \frac{\partial \Lambda}{\partial \varepsilon}, \Lambda \frac{\partial n e f f}{\partial T}+n e f f \frac{\partial \Lambda}{\partial T}\right]\left[\begin{array}{l}
\Delta \varepsilon \\
\Delta T
\end{array}\right],
$$

The first term of the row matrix stands for the strain dependency while the second term shows the temperature dependence of the $\lambda_{B}$ variation. In this matrix equation, the $\partial \mathrm{T}$ and $\partial \varepsilon$ are small temperature and strain alteration, respectively. According to equation (2), in case of pure strain sensor, temperature is not perturbed $\partial \mathrm{T}=0$, and the Bragg wavelength shift will depend only on the external strain $\partial \varepsilon$ that involves two components. The first component shows the change of refractive index due to the strain because of photo elastic effect and the second component shows physical elongation because of Poisson effect.

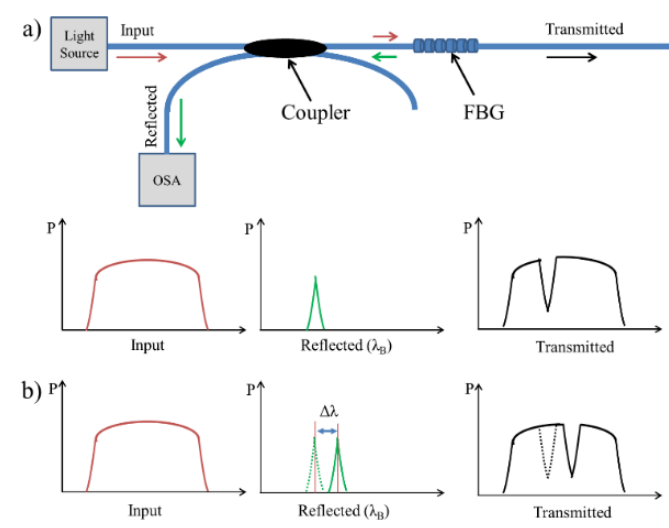

Figure 3: Principle of FBG performance.

\section{Initial FBG Structure for Strain Sensor}

In this work, in all the developments, a FBG structure with $L$ $=20 \mathrm{~mm}$ is considered. The initial structure is assumed to be a standard telecommunication single-mode step index optical fiber that is inscribed with two parameters, core radius $(a)$ and defined by:

$$
\Delta=\frac{n_{\text {core }}^{2}-n_{\text {clad }}^{2}}{2 n_{\text {clad }}^{2}}
$$

Initial values are set at $\Delta=0.0018$ and $\mathrm{a}=4 \mu \mathrm{m}$. In the absence of external strain, the Bragg wavelength is adjusted at 1550 $\mathrm{nm}$ through management of geometrical and optical features of periodic grating structure. The grating part contained no chirp, no apodization and index modulation is 0.0006 . The reflection responses of initial structure with no strain and under 1000 micro external strain are shown in figure 4 . The simulation results show that the applied strain only caused the reflection response shifted to the higher wavelengths with sensitivity of $1.2 \mathrm{pm}$ per micro strain and have no considerable effect on the side lobes and also the main lobe kept nearly $100 \%$ of reflected light power.

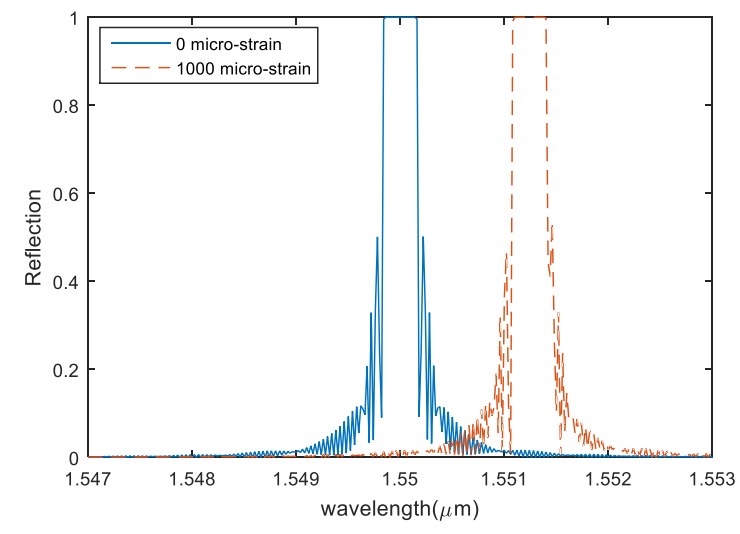

Figure 4: Reflection response of FBG initial structure as a function of applied strain.

\section{Grating and Optical Parameters of FBG Sensor Structure Consideration}

The refractive index profile of the grating can be modified through addition of other features such as chirp, apodization, index modulation, grating length and core index and width to achieve better results. The effect of these grating and optical structure parameters are studied on the reflection response in terms of side lobes, main lobe power and strain sensitivity of Bragg wavelength. The side lobes are due to multiple reflections at the grating ends. Then the parameters are adjusted for better performance. In simultaneous presentation of the reflection and sensitivity in simulations, the induction of 1000 micro-strain is considered.

Any variation in the grating period along the fiber length is called chirp. Based on the simulation results exhibited in figure 5, monitoring on chirp parameter can attenuate side lobes to give better result for the reflected main power although decreases magnitude of main lobe power and increases its bandwidth. It is evident that the applied chirp 
has no effect on Bragg wavelength and hence no improvement is achieved in sensitivity.

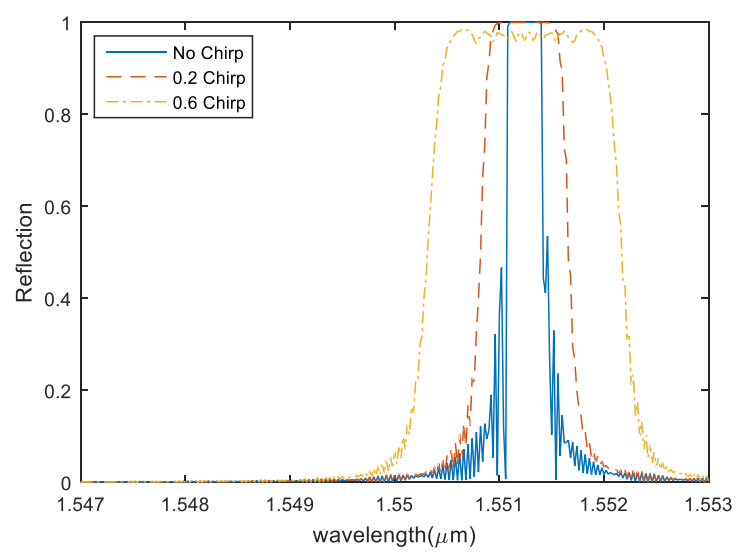

Figure 5: Reflection response in FBG with different chirp under 1000 micro strain.

Apodization is a variation of the modulation depth along the grating length $[10,11]$. The apodized fiber bragg grating plays an important role to suppress the side lobes while maintaining a high percentage of reflectivity and narrow bandwidth. Apodization techniques with defined profiles, generally as verified in figure 6, suppress side lobes and provide better performance for the structure but does not change the Bragg wavelength. Gaussian and Super-gaussian profiles give much more effective suppression behavior than the tan. one. Tan. and Super-gaussian profiles keep $100 \%$ of main lobe power but Gaussian profile couldn't. Therefore, concluding that the Super-gaussian profile is capable of adjusting the best reflection response for the structure. From strain sensitivity point of view, it is obvious that the grating refractive index apodization has no remarkable effect. In other words, the apodization could not change the FBG sensor sensitivity to applied external strain.

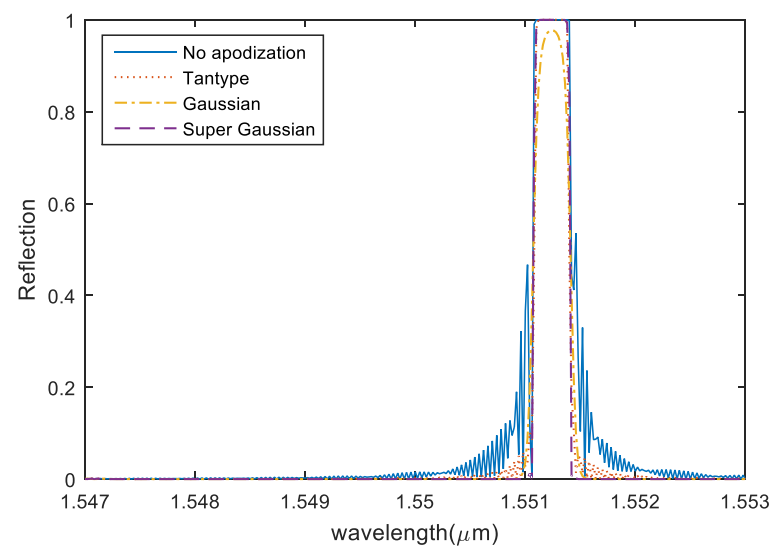

Figure 6: Reflection response of FBG with three different apodization under 1000 micro strain.

Different typical values are examined for index modulation in this work. The results for four values are shown in figure 7. Reduction in index modulation decreases the reflection bandwidth as well as reducing side lobes by trading off losing main lobe power. As a result a proper moderate value should be selected for index parameter. Also, it is clear that there is no effect on strain sensitivity owing to index modulation variation. Bragg wavelength is the same in all of diagrams for all index values.

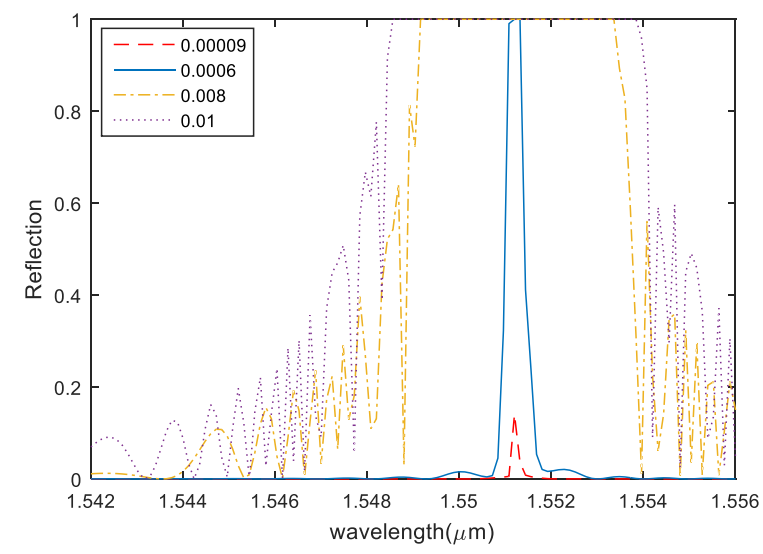

Figure 7: Reflection response in FBG with different index modulation under 1000 micro strain.

Grating length by two values $20 \mathrm{~mm}$ and $30 \mathrm{~mm}$ are tested. The results in figure 8 show that the increase in grating length can weakly affect side lobes of the reflection response and does not lead to any variation in sensitivity on Bragg wavelength under strain application.

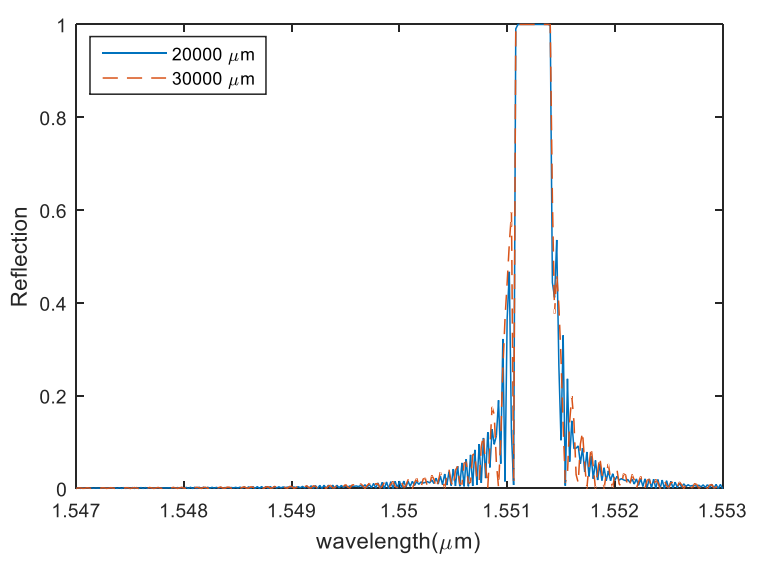

Figure 8: Reflection response in FBG with different grating length under 1000 micro strain.

Based on the exhibited results, admittedly, grating structure parameters affect only the reflection response and do not have any impact on Bragg wavelength sensitivity to strain.

In the following, the optical and geometrical parameters of the optical fiber which the grating structure is placed in its core are investigated. As stated before, the initial values of the core radius $(a)$ and $\Delta$ are $4 \mu m$ and 0.0018 .

At first, the effect of fiber refractive index difference ( ) on the strain sensitivity of the FBG structure is studied and presented in figure 9. The results admit that while the core 
index increases, the sensitivity to strain is decreased by moving the main lobe towards left. Likewise, as the core index falls, there is a right hand shift in main lobe position which means that the sensitivity is boosted. In addition, the side lobs are reduced as the core index is reduced. In the meantime, any variation in the $\Delta$ parameter has no effect on the $\lambda_{\mathrm{B}}$ value under normal condition. So extra shift of the $\lambda_{\mathrm{B}}$ in the $1000 \mu \varepsilon$ application is only owing to strain sensitivity growth of the structure.

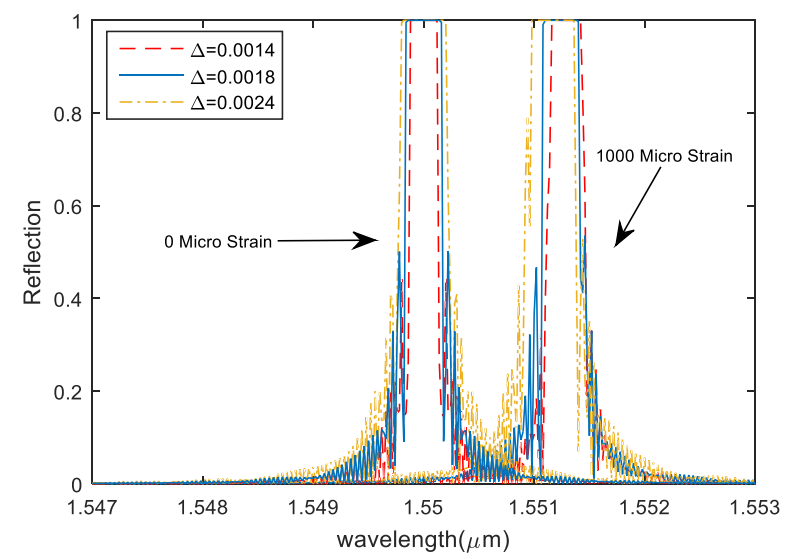

Figure 9: Reflection response in FBG as a function of refractive index difference under 0 and 1000 micro strain.

The reflection response is presented for several core radiuses as shown in figure 10. Decrement in the core radius shifts the $\lambda_{\mathrm{B}}$ toward higher wavelength under 1000 micro strain application. This means that the structure sensitivity to strain is boosted by core radius decrement. Meanwhile, the main lobe reflection power is decreased so much. The core radius increment decreases the strain sensitivity and results the larger bandwidth and side lobes in the reflection response. At zero strain circumstances, there is no shift for the Bragg wavelength versus the core radius changes.

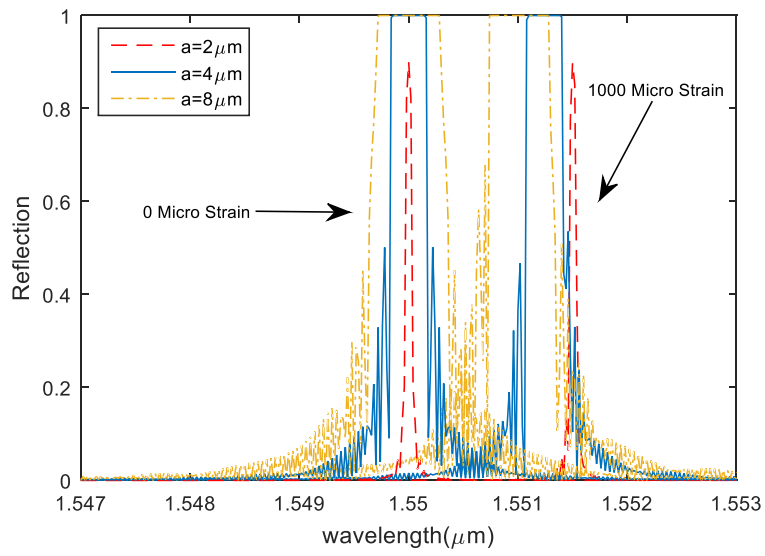

Figure 10: Reflection response in FBG as a function of core radius under 0 and 1000 micro strain.
One of the main problems of designed sensors is side sensitivities particularly to temperature. The side sensitivity of a sensor, being sensitive to undesirable environmental parameters, leads to measurement errors [9].

The simulation result in figure 11 shows the effect of temperature variation on the Bragg wavelength shift from $1.55 \mu \mathrm{m}$ in the absence of strain application. The initial thermal condition of design is assumed to be $25^{\circ} \mathrm{C}$, and the Bragg wavelength shift is zero at this temperature. The Bragg wavelength moves to higher values with temperature increment such that Bragg wavelength shift at $37^{\circ} \mathrm{C}$ according to figure 11 is $156 \mathrm{pm}$ with temperature sensitivity of $13 \mathrm{pmC}^{-1}$. Since it is aimed to employ the FBG sensor in vivo, the Bragg wavelength must be readjusted at $37^{\circ} \mathrm{C}$ in $1.55 \mu \mathrm{m}$. Therefore, the required removal offset at $37^{\circ} \mathrm{C}$ should be $156 \mathrm{pm}$. This can be done by offsetting the Bragg wavelength at 25 degree with $-156 \mathrm{pm}$ only once by alteration a little bit in grating index change parameter that does not affect sensitivity as shown in figure 12 .

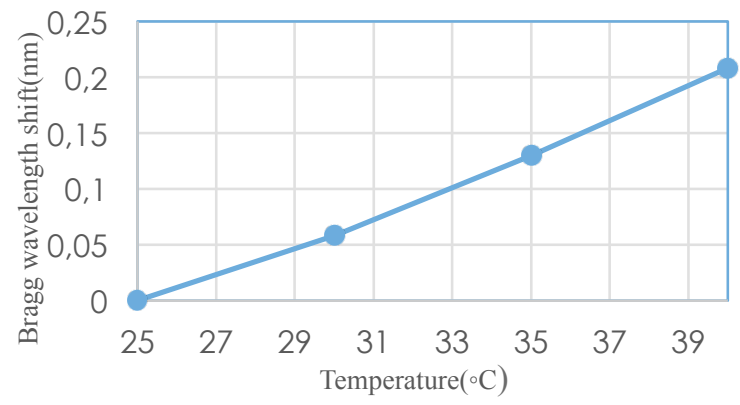

Figure 11: Undesirable Bragg wavelength shift (nm) versus temperature $\left({ }^{\circ} \mathrm{C}\right)$.

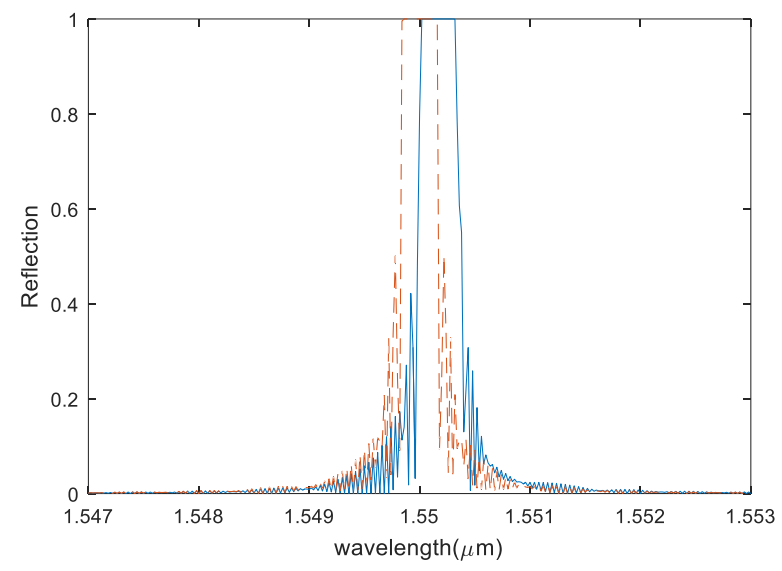

Figure 12: Initial structure (solid line) and structure with temperature offset removal (dash line) reflection response at $37^{\circ} \mathrm{C}$. 


\section{Discussion and Proposed FBG Sensor}

Attained results in section 4 clearly indicate that the reduction in the refractive index difference ( ) and core radius (a) improve sensitivity efficiently. Thus, it is endeavored to decrease the both parameters to obtain high sensitivity. The concern to considerate is that it might cause main lobe losses a little power to side lobes in this condition. To overcome this drawback, it is concluded from previous section that through applying reasonable chirp and using apodization technique with Super-gaussian profile and also increasing of index modulation we can managed to suppress side lobes and powering of main lobe without affecting sensitivity. Clearly optimization process has been done in two step. At first step modified geometrical and optical parameters of optical fiber structure leads to maximize observed $\lambda_{B}$ shift under strain. In the second step best reflection response for maximized $\lambda_{B}$ shift obtained in first step is achieved by modifying grating parameters. Finally, the best values, based on the demonstrated discussions, are selected for grating parameters to obtain a modified FBG sensor structure for further development. The characteristics of the proposed structure are presented in table 1. Desirable reflection response with the high sensitivity $1.54 \mathrm{pm} \mu \varepsilon^{-1}$ obtained with no side lobe and nearly $100 \%$ light power for main lobe shown in figure 13.

Table 1: Optimised value for FBG structure parameters.

\begin{tabular}{|l|l|}
\hline Parameter & Value \\
\hline$\Delta$ & 0.0014 \\
\hline Core radius & $2 \mu \mathrm{m}$ \\
\hline$\lambda_{B}$ & $1550 \mathrm{~nm}$ \\
\hline Index Change & -0.012 \\
\hline Chirp & 0.08 \\
\hline Apodization & Super-Gaussian \\
\hline Length( $\mu \mathrm{m})$ & \\
\hline Index Modulation & 0.02 \\
\hline
\end{tabular}

Applying different strains to proposed sensor admit the linear behavior for sensor as clarified in figure 14 . Obviously sensor installation with the experimental setup including sources, detectors, OSA, power supply and different displays that are conventionally used in vivo application involve challenges that we should investigate and find proper solution.

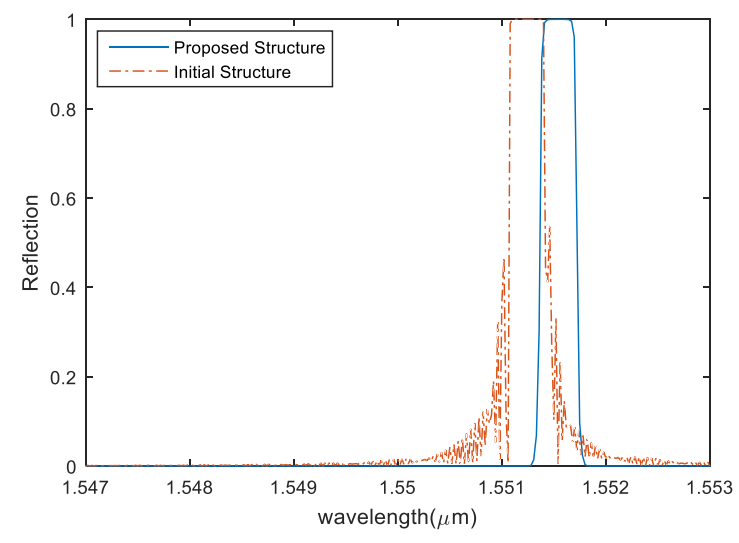

Figure 13: High sensitivity in reflection response of proposed FBG strain sensor at 37 degree Celsius and 1000 micro-strain.

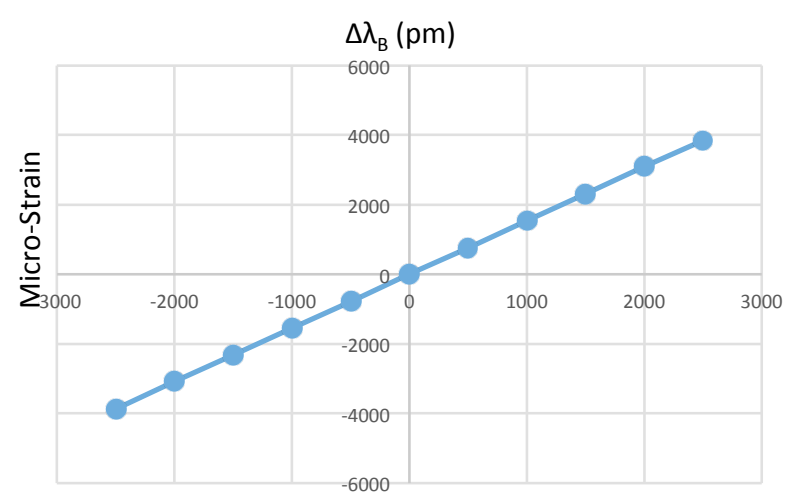

Figure 14: Linearity in proposed FBG strain sensor (Left side: Compressive, Right side: Tensile).

\section{Conclusion}

In this article, an initial structure based on standard singlemode step index optical fiber is selected for muscular FBG strain sensor. The performance of structure has been optimised in terms of side lobe suppression and strain sensitivity for different grating and structure parameters. Simulation outcomes admit that the structure strain sensitivity is enhanced by adjusting the optical fiber geometrical and optical parameters. The modified structure provides excellent reflection response with minimum power loss by suppression of side lobes and detection of small strain changes. The achieved $1.54 \mathrm{pm} \mu \varepsilon^{-1}$ sensitivity is almost 30\% higher than the well-known sensitivity of 1.2 $\mathrm{pm} \mu \varepsilon^{-1}$ reported for FBGs. This remarkable result is clearly obtained from simulation of sensor structure. That would be better to evaluated experimentally as well. 


\section{References}

[1] C. Milgrom, A. Finestone, A. Simkin, I. Ekenman, S. Mendelson, M. Millgram, M. Nyska, E. Larsson, D. Burr, In vivo strain measurements to evaluate the strengthening potential of exercises on the tibial bone, Journal of Bone Joint Surg [Br] 82-B: 591-594, 2000

[2] F. Makouie, and S. Makouie, Efficient optical method for musuloskeletetal strain monitoring in physical activities, International Journal of Engineering and Applied Sience 7: 1-5, 2015.

[3] F. Urban, J. Kadlee, R. Vlach, and R. Kuchta, Design of a pressure sensor based on optical fiber bragg grating lateral deformation, Sensors 10: 11212-11225, 2010.

[4] E. Li, J. Xi, J.F. Chicharo, and Y. Zhang, The experimental evaluation of FBG sensor for strain measurement of prestressed steel strand, Proceeding of SPIE 5649, 2005.

[5] Z. Zhou, T. W. Graver, L. Hsu, J. Ou, Advanced FBG sensors, fabrication, demodulation, encapsulation and the structural health monitoring of bridges, Pacific Science Review 5: 116-121, 2003.

[6] P. Roiz, L. Carvalho, O. Frazao, J.L. Santos, and J.A. Simoes, From conventional sensors to fiber optic sensors for strain and force Measurements in Biomechanics Application: a review, journal of Biomechanics 47: 1251-1261, 2014.

[7] A.G. Mignani and F. Baldini, Biomedical sensors using optical fibers, Reports on Progress in Physics 59: 1-28, 1996.

[8] S. Ju, P.R. Watekar and W.T. Han, Enhanced Sensitive of the FBG Temperature Sensor Based on the PbO$\mathrm{GeO}_{2}-\mathrm{SiO}_{2}$ Glass Optical Fiber, Journal of Lightwave Technology 28: 2697-2700, 2010.

[9] F. Makouei, S. Makouei, GA based management of strain response in RII-type single mode optical fiber, Optik 127: 8333-8340, 2016.

[10] I. Yullianti, A.S.M. Supa'at, and A.M. Al-hetar, Simulation of apodization profiles performances for unchirped fiber Bragg gratings, IEEE Intern. Conf. on Photonics (ICP): 1-5, 2015.

[11] Sher Shermin A. Khan and Md. S. Islam, Determination of the Best Apodization Function and Grating for Dispersion Compensation, Journal of Communication 7: 840-847, 2012. 